\title{
Combining Probability Scores to Optimize Clinical Use of the NICHD Neonatal BPD Outcome Estimator
}

Rebekah M. Leigh, John B. C. Tan, Shirin R. DeGiorgio, Minha

Cha, Chelsea Kent, Hung-Wen Yeh, Fu-Sheng Chou

\begin{abstract}
Objective: Bronchopulmonary dysplasia (BPD) continues to prevail among very preterm infants. While NICHD BPD Outcome Estimator is easy to use, the clinical interpretation remains challenging. This study aims to optimize its use.

Study Design: A retrospective study was conducted with 469 infants born between 2015 and 2020. Data were entered into the Estimator to obtain probability scores. Trajectories of the probability scores were modeled using generalized additive modeling. The optimal cutoff number for predicting severe BPD or death was identified by a grid search from a range established by the original population distribution and the ROC curve.
\end{abstract}

Result: Combining probability scores from the severe and death categories and the no-BPD and mild categories may improve BPD outcome prediction. A cutoff of $21 \%$ combining outcome probabilities from severe and death categories is predictive of severe BPD or death.

Conclusion: Combining probability scores of different categories improves BPD outcome prediction.

\section{Keywords}

Bronchopulmonary dysplasia, Neonatal BPD Outcome Estimator, generalized additive modeling

\section{"Among the survivors, bronchopulmonary dysplasia (BPD) is the most common respiratory complication of extreme prematurity (less than 28 weeks of gestation) and affects one-third or more of preterm infants (5). Despite improvement in other morbidities of extreme prematurity, the incidence of BPD remains high $(6,7) . "$}

\section{Introduction}

Preterm birth contributes significantly to both neonatal morbidity and mortality. Vast improvement in the preterm mortality rate due to advancements in neonatal care over the years has been at the expense of these infants who experience increased adverse outcomes such as respiratory illness, neurodevelopmental delay, and behavioral problems, among many others (1-4). Among the survivors, bronchopulmonary dysplasia (BPD) is the most common respiratory complication of extreme prematurity (less than
28 weeks of gestation) and affects one-third or more of preterm infants (5). Despite improvement in other morbidities of extreme prematurity, the incidence of BPD remains high $(6,7)$.

BPD is a multifactorial disease that results from a vulnerable, underdeveloped lung experiencing injury, inflammation, and oxidative stress causing prolonged oxygen dependence, a lengthened hospital stay, and significant long-term pulmonary morbidities that may continue into adulthood (8-10). BPD occurs due to disrupted postnatal lung development with alveolar simplification and dysmorphic microvascular changes (11). The interruption of postnatal alveolarization decreases gas exchange in the lung and elevates the requirement for supplemental oxygen or mechanical ventilation, which can exacerbate the existing lung damage and inflammation (12). Due to the improvement in mortality rates of preterm neonates, the research focus for BPD has shifted to reducing the burden of the disease by promoting postnatal alveolarization and facilitating recovery through therapies such as optimized ventilation, surfactant replacement, caffeine, antioxidants, vitamin A, and corticosteroids (13-15).

"Corticosteroids, in particular, have shown numerous benefits in the de-escalation of respiratory support by reducing lung inflammation and improving the exchange rate of gases, although its role in BPD prevention is currently unclear $(16,17)$. However, systemic corticosteroid use has also been associated with impaired neurodevelopment, cerebral palsy, increased risk of intestinal perforation with indomethacin exposure, and late-onset sepsis $(18,19)$."

Corticosteroids, in particular, have shown numerous benefits in the de-escalation of respiratory support by reducing lung inflammation and improving the exchange rate of gases, although its role in BPD prevention is currently unclear $(16,17)$. However, systemic corticosteroid use has also been associated with impaired neurodevelopment, cerebral palsy, increased risk of intestinal perforation with indomethacin exposure, and late-onset sepsis $(18,19)$. Given the potential for both benefit and harm, the prudent use of corticosteroids is suggested for infants when the severity risk of BPD is considerable $(>50 \%)(20,21)$. One method to guide corticosteroid intervention is by using the Eunice Kennedy Shriver National Institute of Children Health and Human Development (NICHD) Neonatal BPD Outcome Estimator, developed in 2011, to quantify the risk (22). The model predicts four BPD outcomes (no BPD, mild, moderate, severe) or death based on demographic 
variables and respiratory support for one postnatal day and is restricted by range limits for birth weight, gestational age, and race/ ethnicity (only applicable for white, black, and Hispanic) (22). The Estimator is an easily accessible tool (23) for identifying infants with a risk of high severity BPD who may significantly benefit from postnatal corticosteroids.

A systematic review and meta-analysis showed that the Estimator is currently the best prediction tool for BPD (24). Yet, there is no clear consensus on how the Estimator should be used clinically. Recently, Baud et al. further validated the Estimator externally using a French cohort and attempted to optimize the model by introducing three additional variables - respiratory support at baseline, center effect, and multiple pregnancies (25). While the original and the modified models both provide plausible $C$ statistics results, the evaluation of each model's accuracy, based on an optimal probability cutoff for each severity category, has not been extensively reported. Furthermore, Cuna et al. showed that a combined probability score of severe or death ( $>37 \%$ ) along with a no BPD probability score $(<3 \%)$ on postnatal day 14 was predictive of systemic corticosteroid administration, suggesting that the probability scores may be used independently or in combination (26). Since the study still did not provide a cutoff value for BPD outcome prediction, it remains unclear whether the severity category bearing the highest probability score should be considered the predicted BPD outcome or whether probability scores from different severity categories should be combined for clinical use.

\section{"This study investigated how to translate the probabilities for each BPD category best to be clinically informative. We hypothesize that a comprehensive understanding of the probability trajectories for each BPD severity category is crucial to developing an optimal methodology for interpreting the results of the Estimator."}

This study investigated how to translate the probabilities for each BPD category best to be clinically informative. We hypothesize that a comprehensive understanding of the probability trajectories for each BPD severity category is crucial to developing an optimal methodology for interpreting the results of the Estimator.

\section{Subjects and Methods}

\section{Study population and data collection}

This observational retrospective study was approved by Loma Linda University Institutional Review Board with a waiver for informed consent. Preterm infants included in the study were born at 30 weeks gestational age (GA) or less with a race/ethnicity designated as white, black, or Hispanic. All infants were admitted to the neonatal intensive care unit (NICU) at the Loma Linda University Children's Hospital between 2015 and 2020 with respiratory data available at postnatal days 1, 3, 7, 14, 21, and/or 28 and at 36 weeks' CGA for BPD outcome assessment. The categories of race/ethnicity and the discretization of the postnatal days are limitations set by the Estimator.

Demographic data including sex, race/ethnicity, birth weight, gestational age at birth, mortality before 36 weeks' CGA, as well as clinical data such as antenatal steroids, mode of delivery, postnatal steroids, and patent ductus arteriosus treatment, were collected with chart review. Notably, antenatal steroids were considered as administered if the mother received at least one dose prior to delivery. Additionally, we defined postnatal steroids (i.e., dexamethasone) as prescribed for BPD if given for a full 10-day course or more.

Other demographic information including birth GA, birth weight, sex, race/ethnicity, and respiratory data including ventilator type and oxygen fraction $\left(\mathrm{FiO}_{2}\right)$ on six postnatal days $(1,3,7,14,21$, and 28), when available, were obtained from the infants' medical charts. If a birth weight fell out of the range required by the Estimator (which differs for each GA), the closest weight suggested by the tool was used. Based on the options available in the Estimator, ventilator types were categorized into (1) high-frequency ventilation, which included high-frequency oscillator and high-frequency jet ventilator; (2) IMVISIMV, which included conventional tidal ventilators; (3) continuous positive airway pressure (CPAP), which included regular CPAP or non-invasive positive pressure ventilation (NIPPV); (4) cannula/hood, which included either high-flow and low-flow nasal cannula. After entering the demographic and respiratory data into the Estimator, the probability scores for each BPD severity category were generated and recorded for the trajectory model development and validation.

\section{$B P D$ clinical outcome designation}

The 2001 definition of BPD severity category was used to categorize respiratory outcomes, in which respiratory support of fewer than 28 days was defined as no BPD; respiratory support of more than 28 days with no need for respiratory support at 36 weeks CGA was defined as mild; respiratory support of more than 28 days with continued support at 36 weeks CGA by nasal cannula with $\mathrm{FiO}_{2}<30 \%$ was considered moderate, and respiratory support of more than 28 days with support at 36 weeks by nasal cannula with $\mathrm{FiO}_{2} \geq 30 \%$ or by positive pressure support was defined as severe BPD (13). Infants meeting the inclusion criteria but died before reaching 36 weeks CGA were included in the death category.

\section{Severity probability score trajectory model development}

Upon initial assessment of the probability score trajectories for each severity category, a non-linear trend was noted. Additionally, given repeated measurement of ventilator type and $\mathrm{FiO} 2$ across postnatal days of each infant, we utilized a generalized additive mixed modeling (GAMM) algorithm to model trajectories of the probability scores. In the initial model, we included the five severity categories of the outcome probabilities for each of the five clinical outcomes (severity-by-outcome, 25 levels) as fixed-effect; we also included a smoothed function of the postnatal day alongside its interaction with severity-by-outcome. In the subsequent models, the levels of the severity-by-outcome variable were reduced to 10 levels encompassing two severity categories for each of the five clinical outcomes or to 2 levels encompassing one severity category for dichotomized clinical outcome groups. The residual term distribution was assessed to ensure model adequacy. Generalized cross-validation was performed during the fitting process. 
Table 1. Patient characteristics.

\begin{tabular}{|c|c|c|c|}
\hline $\mathrm{P}_{\text {Variable }}^{\text {BPD Group }}$ & No BPD/Mild/Moderate & Severe/Death & $P$ value \\
\hline Number of patients & 220 & 249 & - \\
\hline Female & $121(55 \%)$ & $100(40.2 \%)$ & 0.002 \\
\hline Race/ethnicity & & & \multirow{4}{*}{0.44} \\
\hline White & 51 & 46 & \\
\hline Black & 41 & 47 & \\
\hline Hispanic & 128 & 156 & \\
\hline Median gestational age & 27 week 6 days & 25 week 4 days & $<0.001$ \\
\hline Mean birth weight & $950 \pm 186$ grams & $774 \pm 186$ grams & $<0.001$ \\
\hline Death before 36 weeks CGA & 0 & 53 & $<0.001$ \\
\hline Antenatal steroids & & & 0.29 \\
\hline Yes & 202 & 218 & \\
\hline No & 16 & 26 & \\
\hline Unknown & 2 & 5 & \\
\hline C-section & $157(79 \%)$ & $174(70 \%)$ & 0.80 \\
\hline Postnatal steroids & 8 & 76 & $<0.001$ \\
\hline Postnatal steroids $\leq 28$ days of life & 6 & 32 & $<0.001$ \\
\hline \multicolumn{4}{|l|}{ Patent ductus arteriosus } \\
\hline Medical treatment & 26 & 31 & 0.95 \\
\hline Surgical Ligation & 12 & 54 & \multirow{2}{*}{$<0.001$} \\
\hline Embolization & 2 & 8 & \\
\hline Number of training data points & 1,200 & 984 & - \\
\hline Number of testing data points & 58 & 191 & - \\
\hline
\end{tabular}


Clinical BPD outcome groups
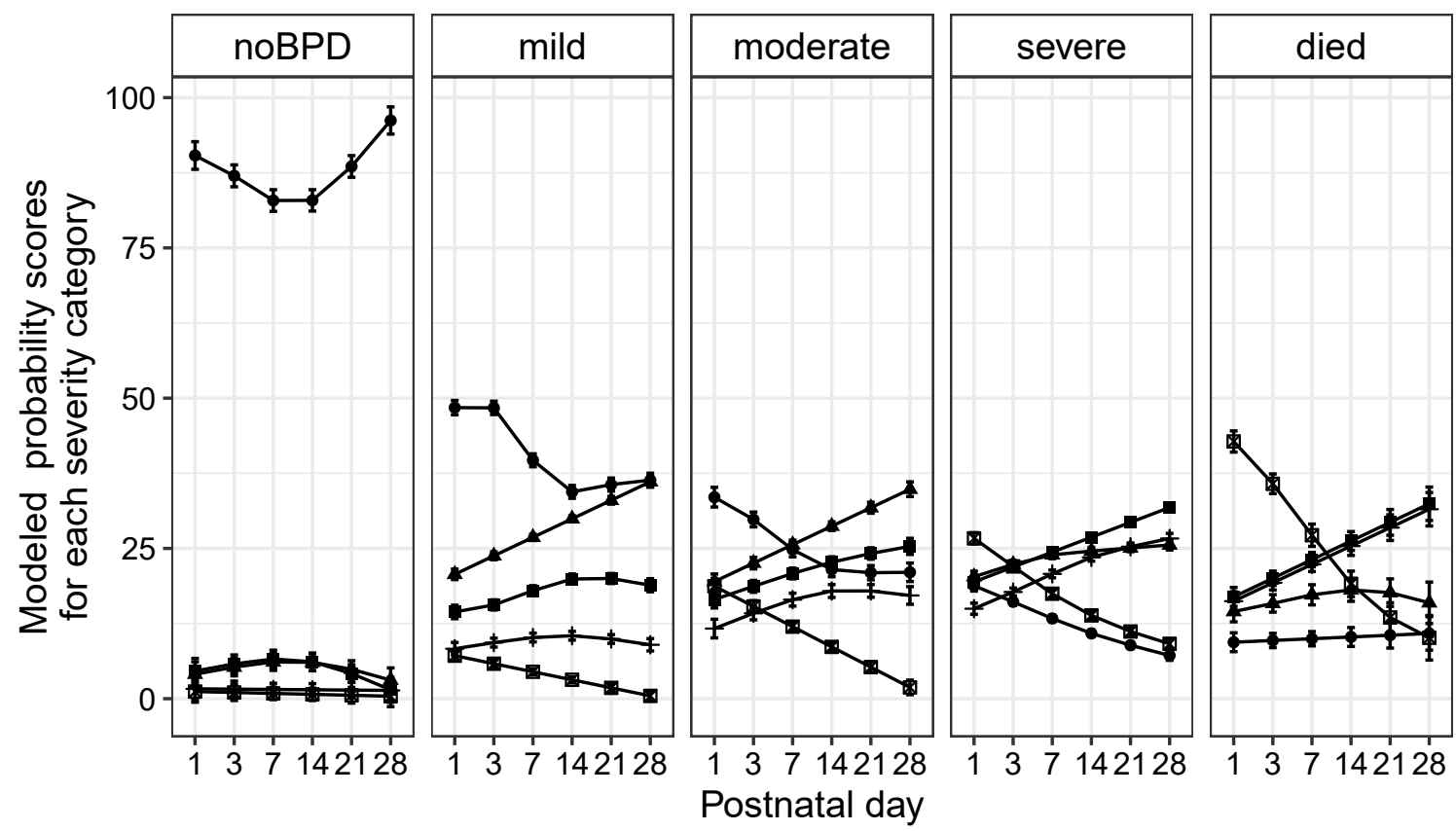

Severity category

- NoBPD

\ Mild

- Moderate

+ Severe

$\bowtie \quad$ Death

Figure 1. Trajectories of probability scores of each severity category for each clinical BPD outcome group. A plot for the modeled trajectories of the probabilities of each severity category for each postnatal day stratified by clinical BPD outcome. Dots represent predicted values; error bars represent $95 \%$ confidence intervals.

Clinical BPD outcome groups
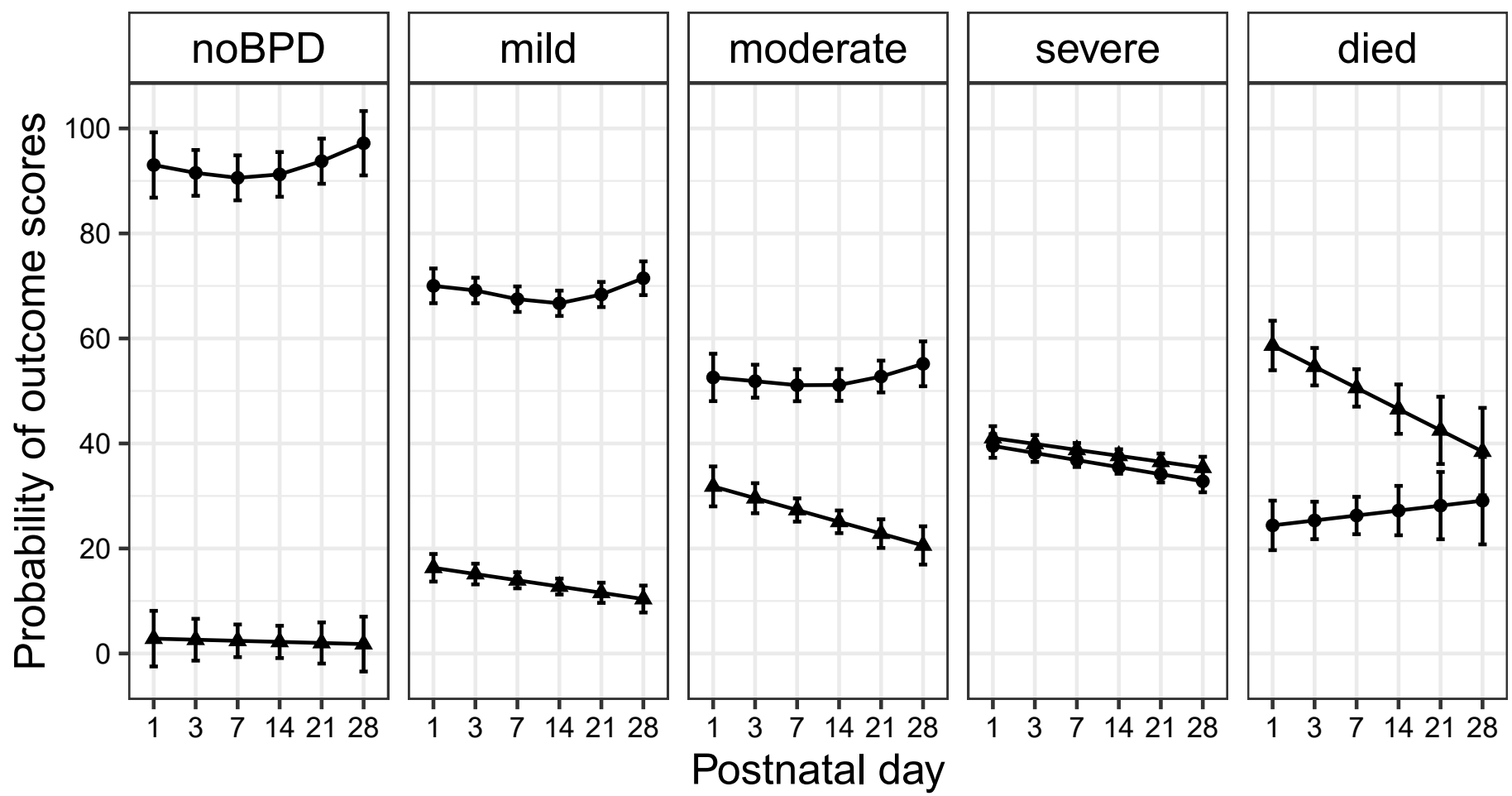

\section{Scores - Combining No BPD and Mild $\Delta$ Combining Severe and Death}

Figure 2. Trajectories of combined probability scores for each clinical BPD outcome group. A plot for the modeled trajectories of the probabilities of each postnatal day stratified by clinical BPD outcome following combining the probability scores for no BPD and mild categories as well as severe illness and death categories. Dots represent predicted values; error bars represent $95 \%$ confidence intervals. 


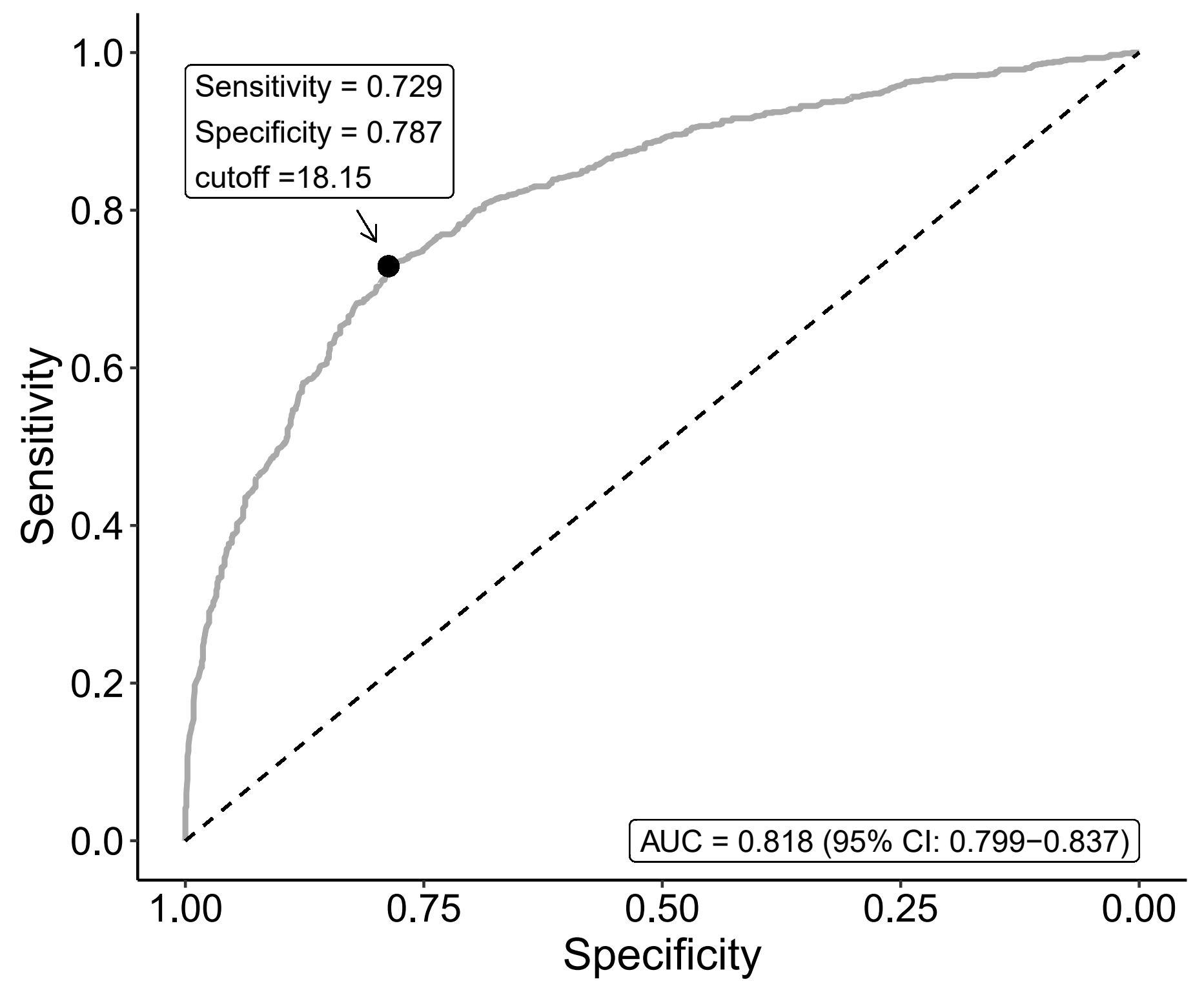

Figure 3. A receiver's operating characteristic curve shows the BPD outcome's predictability using combined probability scores of severe disease and death. The curve showed the relationship between sensitivity and specificity using various cutoff points for the combined probability scores. The area under the curve (AUC) was 0.818 (95\% confidence interval: 0.799-0.837). A Youden's J statistics showed a cutoff of 18.15 provides the most balanced sensitivity and specificity.

The final model equation is as follows:

Probability score = severity-by-outcome + smoothed (postnatal day, by = severity-by-outcome)

\section{Statistical analysis and performance assessment}

Descriptive statistics were performed to characterize the patient population. Student's $t$-test or Wilcoxon rank-sum test was used for continuous variables, and chi-squared test was used for the categorical variables.

For binary outcome prediction, a confusion matrix was used. Sensitivity, specificity, positive predictive value (PPV), negative predictive value (NPV), and overall accuracy were calculated. Additionally, the area under the receiver's operating characteristic (auROC) curve was calculated to assess the predictability of respiratory outcome using combined probabilities from the Estima- tor.

All statistical analyses were performed in R 4.1.0 using RStudio 1.4 (27). The GAMM models were developed using the gamm4 package (28). Codes are available upon request.

\section{Results}

Infant characteristics

We identified 469 infants from 2015 to 2020, meeting inclusion criteria with a total of 2,433 respiratory data points (infants born at outside hospitals may not have respiratory data prior to transfer for probability score calculation). Detailed patient characteristics are available in Table 1. Out of the 469 infants, 220 with no BPD, mild, or moderate were categorized as "non-severe," whereas the other 249 infants were categorized in the "severe" group. Fiftyfive percent of the infants in the non-severe group were female, 


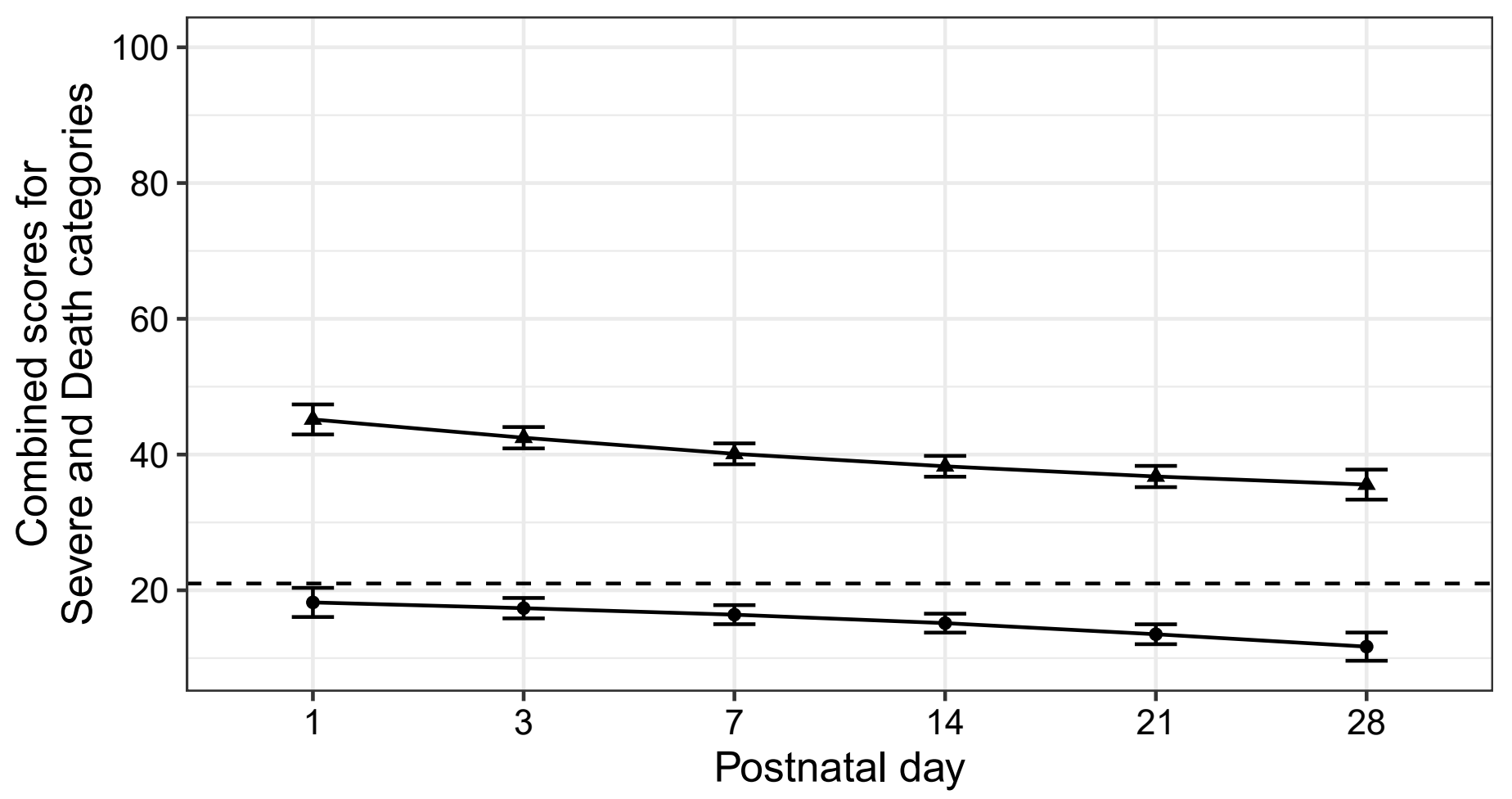

\section{Clinical BPD outcome groups}

- non-severe (no, mild, or moderate BPD)

- severe (severe BPD or died)

Figure 4. Trajectories of combined probability scores for dichotomized clinical BPD outcome groups. A plot for the modeled trajectories of the probabilities of each postnatal day following combining the probability scores for severe and death categories and dichotomizing the clinical BPD outcome groups into the non-severe (no, mild or moderate BPD outcome) group and the severe (severe BPD or died before 36 weeks' corrected gestational age) group. Dots represent predicted values; error bars represent $95 \%$ confidence intervals. The horizontal dashed line represents the cutoff number of 21.

compared to $40 \%$ in the severe group. The mean GA in the nonsevere group was 27 weeks 5 days \pm 1 week 6 days, compared to 25 weeks 6 days \pm 1 week 6 days in the severe group $(p<0.001$, Table 1).

Additionally, the mean birth weight was $950 \pm 186$ grams in the non-severe group, compared to $774 \pm 186$ grams in the severe group $(p<0.001)$. There was no significant difference between the rate of antenatal steroids and the mode of delivery between the two groups. Infants in the severe group were significantly more likely to receive corticosteroid intervention and invasive intervention for patent ductus arteriosus (PDA). Demographic and clinical comparisons are available in Table 1.

Performance of the Estimator using the highest score of the probability of an outcome for BPD outcome prediction

We first assessed the Estimator's performance by using the maximum probability scores to predict BPD severity. The prediction was assigned by the severity category with the highest probability of outcome score. A five-by-five confusion matrix was constructed (Table 2) and showed a poor correlation between clinical BPD outcome and predicted outcome, with an overall accuracy rate of $29 \%$.

Generalized additive modeling of the trajectories of the probability of outcome scores

All 2,433 sets of probability score data from 469 infants, including $31,125,64,189$, and 60 infants in the no BPD, mild, moderate, severe, and death group, respectively, were used to develop the trajectory model. The modeled trajectories for each respiratory outcome group are shown in Figure 1. Each column represents a clinical BPD outcome or death. Every curve in each clinical outcome represents a trajectory of the probabilities for one severity category over time. The no BPD clinical outcome group showed a distinct predicted probability curve for the no BPD severity category with predicted values all above 80 . However, in the clinical outcome groups of mild, moderate, severe, and death, the trajectories for each severity category demonstrated increasing overlapping as the clinical outcome severity increased. The modeled trajectories of the outcome probabilities confirmed that using the highest probability score to predict clinical BPD outcome is inadequate other than for the no-BPD group.

Combining probability of outcome scores from different severity 


\begin{tabular}{|l|l|l|l|l|l|}
\hline \multicolumn{1}{|c|}{ Clinical outcome } & & & & & \\
Predicted outcome & No BPD & Mild & Moderate & Severe & Death \\
\hline No BPD & 175 & 386 & 108 & 127 & 17 \\
\hline Mild & 1 & 276 & 119 & 311 & 16 \\
\hline Moderate & 1 & 42 & 57 & 265 & 19 \\
\hline Severe & 0 & 7 & 33 & 149 & 19 \\
\hline Death & 0 & 16 & 37 & 188 & 64 \\
\hline
\end{tabular}

Table 2. Comparison between actual clinical and predicted BPD outcome using the NICHD BPD Outcome Estimator probability scores. The numbers shown in the table are the numbers of patients in each corresponding outcome category.

\section{categories}

Infants with severe BPD or who died before 36 weeks' CGA had a higher likelihood of needing invasive treatment for PDA and were more likely to receive systemic corticosteroid treatment, representing infants on the worse end of the spectrum in terms of the respiratory outcome at 36 weeks' CGA. On the other hand, infants with no BPD or mild did not require any respiratory support at 36 weeks' CGA, representing the better end of the spectrum in terms of respiratory outcome. Based on these clinical features, we combined probability scores for severe BPD and death into one score and scores for no BPD and mild into another score, followed by repeating the trajectory modeling of the new combined scores (Figure 2). The predicted trajectories of combined probability scores showed a significantly improved distinction both across the five clinical BPD outcome groups and across the scoring groups (no BPD + mild vs. severe BPD + death). This approach of combining the probability scores represents a better model for predicting clinical BPD outcomes.

\section{Identifying the optimal cutoff threshold to maximize prediction}

Infants predicted to have severe BPD or death are the target population for early interventions, such as providing an aggressive nutrition program, vitamin A injections, and/or systemic corticosteroid administration. The population used for the model development of the Estimator included $26 \%$ of infants, approximately, that had severe BPD or death before 36 weeks' CGA (22). If the distribution of the clinical outcome in our cohort was similar to the distribution of the population used to develop the model, a combined score (severe + death) of 26 would likely be an ideal cutoff. Due to the much higher percentage $(53 \%, 249$ out of 469$)$ of infants in our cohort's severe and death clinical outcome categories, we require a lower cutoff threshold. To further identify the optimal cutoff, the outcome probabilities for both severe BPD and death severity categories were combined, and a ROC curve was generated by comparing the combined probabilities with clinical BPD outcomes of either severe or death (Figure 3). The ROC curve showed an auROC of 0.818 (95\% Cl: $0.799-0.837)$. The optimal cutoff based on Youden's J statistic was $18 \%$ (29). To maximize sensitivity, specificity, PPV, NPV, and accuracy altogether, we performed a grid search between 18 and 26 as cutoff scores for each postnatal day using probability data from $80 \%$ of randomly selected infants. The clinical BPD outcome was dichotomized into a non-severe (no, mild, or moderate BPD) group and a severe (severe BPD or death before 36 weeks' CGA) group. After incorporating all postnatal days, we identified a combined score of 21 as the most optimal cutoff score, giving an overall accuracy of approximately $75 \%$. The performance was comparable using data from the remaining $20 \%$ of the infants. The complete characterization of the prediction performance is shown in Table 3. For a visual depiction, Figure 4 showed the trajectories of the combined probability of outcome scores for severe BPD and death for dichotomized clinical outcome groups comparing infants with non-severe (no BPD, mild, or moderate) disease vs. infants with severe disease (severe BPD or death before 36 weeks' CGA).

\section{Discussion}

In this report, we systematically assessed the probability scores calculated by the NICHD BPD Outcome Estimator in 469 very preterm infants. Our analysis suggested that individual probability scores may not be adequate in prediction, but combining probabilities and trending combined probability scores over time may be more informative to clinicians. Using our regional cohort, we further demonstrated that a summed score from the severe and death categories of more than 21 accurately predicted a dichotomized clinical BPD outcome in $75-80 \%$ of infants.

Evidence suggests a developmental origin of BPD (30). Using respiratory data in the first 28 days of postnatal life can be viewed as one way to assess the potential reversibility of the impact of antenatal factors on BPD development and the need for interventions to alter the trajectory of BPD pathology early on in life.

A common goal for BPD Estimator use is to assist clinicians in determining whether systemic corticosteroid administration is justifiable. Studies have shown that systemic corticosteroid use in a group of infants, along with $50 \%$ requiring oxygen therapy at 36 weeks CGA, showed a clinical benefit by reducing mortality or cerebral palsy (31). This cutoff number is similar to the percentage (approximately 46\%) of infants in the moderate, severe, or death outcome categories used to develop the models for the Estimator. In this study, the most optimal cutoff number assessed by the ROC curve (18\%) was much lower than the percentage of 


\begin{tabular}{|c|c|c|c|c|c|c|}
\hline Postnatal do & Day 1 & Day 3 & Day 7 & Day 14 & Day 21 & Day 28 \\
\hline \multicolumn{7}{|c|}{ Exploratory dataset for grid search } \\
\hline Sensitivity & 0.764 & 0.740 & 0.730 & 0.755 & 0.691 & 0.710 \\
\hline Specificity & 0.741 & 0.742 & 0.750 & 0.738 & 0.807 & 0.831 \\
\hline Positive Predictive Value & 0.750 & 0.720 & 0.720 & 0.717 & 0.755 & 0.793 \\
\hline Negative Predictive Value & 0.755 & 0.761 & 0.759 & 0.774 & 0.751 & 0.759 \\
\hline Accuracy & 0.752 & 0.741 & 0.741 & 0.746 & 0.753 & 0.774 \\
\hline \multicolumn{7}{|l|}{ Validation dataset } \\
\hline Sensitivity & 0.805 & 0.825 & 0.833 & 0.884 & 0.810 & 0.744 \\
\hline Specificity & 0.641 & 0.675 & 0.800 & 0.700 & 0.800 & 0.800 \\
\hline Positive Predictive Value & 0.702 & 0.717 & 0.814 & 0.760 & 0.810 & 0.800 \\
\hline Negative Predictive Value & 0.758 & 0.794 & 0.821 & 0.848 & 0.800 & 0.744 \\
\hline Accuracy & 0.725 & 0.750 & 0.817 & 0.795 & 0.805 & 0.771 \\
\hline
\end{tabular}

Table 3. Assessing performance of combined probability scores for BPD severity prediction. Performance of the NICHD Neonatal BPD Outcome Estimator using combined scores of the severe and death categories with a cutoff number of 21, above which predictive of a severe (positive) disease outcome and below which predictive or a non-severe (negative) disease outcome. The actual clinical outcome was dichotomized into the severe (severe BPD or death before 36 weeks' corrected gestational age) group and the non-severe (no, mild or moderate BPD) group for comparison.

infants in the severe and death outcome categories $(26 \%)$ used to develop the Estimator, suggesting that the Estimator may underestimate the severity of BPD in infants in our cohort. In other words, our findings suggest that the infants admitted to our NICU may require higher acuity care and prolonged respiratory support compared to the cohort used to develop and validate the Estimator. We must also acknowledge that our findings may suggest room for improvement in our neonatal respiratory management approaches. One possible improvement would be to develop a geographically localized cutoff number to improve predictability. Baud et al. found a significant influence of a center-related effect in their efforts to improve the predictability of the Estimator (25). Unfortunately, this regional effect becomes diluted in a large cohort.

We recently modeled postnatal weight and weight z-score trajectories of extremely preterm infants using GAM to faithfully summarize weight gain at various stages of development in the NICU (32). Here, we adopted a similar approach and modeled trajectories of outcome probabilities using our institutional data. Trajectory analysis using a mixed modeling approach with interaction terms allows for a better understanding of interrelationships in the overall direction of the propagation of the probability scores across the clinical outcome groups in one model. Unfortunately, the trajectories across various clinical outcome groups, especially in the mild, moderate, and severe BPD groups, was that the relationship among trajectories within each clinical outcome group was resemblant across clinical outcome groups. These findings confirm that using the probability scores directly from the Estimator would not be feasible. A recent report comparing various grouping strategies also suggested that the grouping strategy used for the Estimator may not be clinically informative (33). In this report, the most optimal grouping strategy did not support a correlation between respiratory support needs for more than 28 days and BPD development. Furthermore, respiratory support modality, rather than $\mathrm{FiO}_{2}$, demonstrated a better correlation with BPD outcome.

Multiple antenatal and postnatal non-respiratory factors have been shown to play a significant role in the respiratory outcome and BPD $(25,30)$. The original Estimator does not incorporate these risk factors into the prediction models developed for a single postnatal day. The Estimator only accounts for two respiratory variables: ventilator type and the fraction of inspired oxygen which may change over time. This approach to predictive model development makes the disadvantageous presumption that all factors contributing to BPD development, known or unknown, can be summarized by these two respiratory variables. This assumption inevitably leads to prediction error when these two respiratory factors cannot encapsulate other contributing factors. For example, infants affected by intrauterine growth restriction may perform su- 
perior to those born at the same GA. Yet, they frequently require prolonged respiratory support beyond 36 weeks CGA and are more likely to be diagnosed with BPD (34). On the other hand, significant events (systemic corticosteroid administration, late-onset sepsis, stage 3 necrotizing enterocolitis, among others) that usually occur after 28 postnatal days may alter the trajectory of respiratory support and change the respiratory outcome at 36 weeks CGA. This illustrates why a model that considers these events would improve predictability (25). Future work may focus on incorporating antenatal exposure variables and postnatal comorbidity variables to assess which variables may improve prediction accuracy. Such effort will aid clinical decision-making and provide greater insight and understanding into how non-respiratory factors may affect respiratory outcomes.

Using the new grading system for BPD, a recent report using the Vermont Oxford Network data found a positive correlation between the grades and adverse respiratory outcomes such as supplemental oxygen use after hospital discharge and tracheostomy (35). Moreover, the study also showed that most of the nonrespiratory comorbidities of extreme prematurity were associated with mechanical ventilator use at 36 weeks CGA (Grade 3). Future work could include developing prediction tools for outcomes stratified by respiratory support type at a predefined postnatal age and/ or for long-term respiratory outcomes beyond infancy.

\section{Limitations}

The study is limited by a lack of prospective and external validation. Additionally, the intrinsic limitations of the Estimator, including low temporal resolution (6 time points out of 28 days), the use of only two respiratory variables, and the lack of non-respiratory factors that may impact the respiratory outcomes in the model are also inherited as limitations to this study.

\section{Conclusion}

In this study, we found that the NICHD Outcome Estimator was prone to underestimating the respiratory severity of our preterm infants. A longitudinal approach based on non-linear modeling of the severity probability score trajectories was proposed, which significantly improved prediction accuracy. Our study emphasizes the crucial role of longitudinal assessment of the respiratory trajectory. Future work may include incorporating more detailed respiratory data alongside antenatal and postnatal non-respiratory comorbidity data to improve respiratory outcome prediction further.

\section{References:}

1. Doyle LW, Anderson PJ. Adult outcome of extremely preterm infants. Pediatrics. 2010 Aug;126(2):342-51.

2. McCormick MC, Litt JS, Smith VC, Zupancic JAF. Prematurity: an overview and public health implications. Annu Rev Public Health. 2011;32:367-79.

3. Blencowe $H$, Cousens S, Oestergaard MZ, Chou D, Moller $A-B$, Narwal $R$, et al. National, regional, and worldwide estimates of preterm birth rates in the year 2010 with time trends since 1990 for selected countries: a systematic analysis and implications. Lancet. 2012 Jun 9;379(9832):2162-72.

4. Myrhaug HT, Brurberg KG, Hov L, Markestad T. Survival and Impairment of Extremely Premature Infants: A Metaanalysis. Pediatrics [Internet]. 2019 Feb 1 [cited 2021 Jul 23];143(2). Available from: https://pediatrics.aappublications.org/content/143/2/e20180933
5. Siffel C, Kistler KD, Lewis JFM, Sarda SP. Global incidence of bronchopulmonary dysplasia among extremely preterm infants: a systematic literature review. J Matern Fetal Neonatal Med [Internet]. 2021;34(11). Available from: http://dx.doi. org/10.1080/14767058.2019.1646240

6. Saigal S, Doyle LW. An overview of mortality and sequelae of preterm birth from infancy to adulthood. Lancet. 2008 Jan 19;371(9608):261-9.

7. Stoll BJ, Hansen NI, Bell EF, Walsh MC, Carlo WA, Shankaran S, et al. Trends in Care Practices, Morbidity, and Mortality of Extremely Preterm Neonates, 1993-2012. JAMA. 2015 Sep 8;314(10):1039-51.

8. Vrijlandt EJLE, Gerritsen J, Boezen HM, Grevink RG, Duiverman EJ. Lung function and exercise capacity in young adults born prematurely. Am J Respir Crit Care Med. 2006 Apr 15;173(8):890-6.

9. Greenough A. Long term respiratory outcomes of very premature birth (<32 weeks). Semin Fetal Neonatal Med. 2012 Apr;17(2):73-6.

10. Carraro S, Filippone $M, D a$ Dalt $L$, Ferraro V, Maretti $M$, Bressan S, et al. Bronchopulmonary dysplasia: the earliest and perhaps the longest lasting obstructive lung disease in humans. Early Hum Dev. 2013 Oct;89 Suppl 3:S3-5.

11. Coalson JJ. Pathology of new bronchopulmonary dysplasia. Semin Neonatol. 2003 Feb;8(1):73-81.

12. Bonadies L, Zaramella P, Porzionato A, Perilongo G, Muraca $M$, Baraldi E. Present and Future of Bronchopulmonary Dysplasia. J Clin Med Res [Internet]. 2020;9(5). Available from: http://dx.doi.org/10.3390/jcm9051539

13. Jobe $A H$, Bancalari E. Bronchopulmonary Dysplasia. Am J Respir Crit Care Med. 2001 Jun 1;163(7):1723-9.

14. Baker CD, Alvira CM. Disrupted lung development and bronchopulmonary dysplasia: opportunities for lung repair and regeneration. Curr Opin Pediatr. 2014 Jun;26(3):306-14.

15. Michael Z, Spyropoulos F, Ghanta S, Christou H. Bronchopulmonary Dysplasia: An Update of Current Pharmacologic Therapies and New Approaches. Clin Med Insights Pediatr. 2018 Dec 11;12:1179556518817322.

16. Olaloko O, Mohammed R, Ojha U. Evaluating the use of corticosteroids in preventing and treating bronchopulmonary dysplasia in preterm neonates. Int J Gen Med. $2018 \mathrm{Jul}$ 3;11:265-74.

17. Pasha $A B$, Chen $X-Q$, Zhou G-P. Bronchopulmonary dysplasia: Pathogenesis and treatment. Exp Ther Med. 2018 Dec;16(6):4315-21.

18. Barrington KJ. The adverse neuro-developmental effects of postnatal steroids in the preterm infant: a systematic review of RCTs. BMC Pediatr. 2001 Feb 27;1:1.

19. Shaffer ML, Baud O, Lacaze-Masmonteil T, Peltoniemi OM, Bonsante F, Watterberg KL. Effect of Prophylaxis for Early Adrenal Insufficiency Using Low-Dose Hydrocortisone in Very Preterm Infants: An Individual Patient Data Meta-Analysis. J Pediatr. 2019 Apr;207:136-142.e5.

20. Thébaud B, Goss KN, Laughon M, Whitsett JA, Abman SH, Steinhorn RH, et al. Bronchopulmonary dysplasia. Nat Rev Dis Primers. 2019 Nov 14;5(1):78.

21. Doyle LW, Cheong JL, Ehrenkranz RA, Halliday HL. Late (> 7 days) systemic postnatal corticosteroids for prevention of bronchopulmonary dysplasia in preterm infants. Cochrane Database Syst Rev. 2017 Oct 24;10:CD001145.

22. Laughon MM, Langer JC, Bose CL, Smith PB, Ambalavanan 
$N$, Kennedy KA, et al. Prediction of bronchopulmonary dysplasia by postnatal age in extremely premature infants. Am J Respir Crit Care Med. 2011 Jun 15;183(12):1715-22.

23. Laughon MM, Langer JC, Bose CL, Smith PB, Ambalavanan $N$, Kennedy KA, et al. NICHD Neonatal Research Network Neonatal BPD Outcome Estimator [Internet]. 2011 [cited 2021 May 10]. Available from: https://neonatal.rti.org/index. cfm

24. Onland W, Debray TP, Laughon MM, Miedema M, Cools F, Askie LM, et al. Clinical prediction models for bronchopulmonary dysplasia: a systematic review and external validation study. BMC Pediatr. 2013 Dec 17;13:207.

25. Baud O, Laughon M, Lehert P. Survival without Bronchopulmonary Dysplasia of Extremely Preterm Infants: A Predictive Model at Birth. Neonatology. 2021 May 18;1-9.

26. Cuna A, Liu C, Govindarajan S, Queen M, Dai H, Truog WE. Usefulness of an Online Risk Estimator for Bronchopulmonary Dysplasia in Predicting Corticosteroid Treatment in Infants Born Preterm. J Pediatr. 2018 Jun;197:23-28.e2.

27. $R$ Core Team. R: A Language and Environment for Statistical Computing [Internet]. Vienna, Austria: $R$ Foundation for Statistical Computing; 2018. Available from: https://www.Rproject.org/

28. Wood SN. Stable and efficient multiple smoothing parameter estimation for generalized additive models. J Am Stat Assoc. 2004 Sep;99(467):673-86.

29. Youden WJ. Index for rating diagnostic tests. Cancer. 1950 Jan;3(1):32-5.

30. Morrow $L A$, Wagner BD, Ingram DA, Poindexter BB, Schibler $K$, Cotten CM, et al. Antenatal Determinants of Bronchopulmonary Dysplasia and Late Respiratory Disease in Preterm Infants. Am J Respir Crit Care Med. 2017 Aug 1;196(3):36474.

31. French NP, Hagan R, Evans SF, Mullan A, Newnham JP. Repeated antenatal corticosteroids: Effects on cerebral palsy and childhood behavior. Am J Obstet Gynecol. 2004 Mar 1;190(3):588-95.

32. Chou F-S, Yeh H-W. Sex differences in postnatal weight gain trajectories of extremely preterm newborns. J Perinatol [Internet]. 2021 May 25; Available from: http://dx.doi. org/10.1038/s41372-021-01099-2

33. Jensen EA, Dysart K, Gantz MG, McDonald S, Bamat NA, Keszler M, et al. The Diagnosis of Bronchopulmonary Dysplasia in Very Preterm Infants. An Evidence-based Approach. Am J Respir Crit Care Med. 2019 Sep 15;200(6):751-9.

34. Gortner L, Reiss I, Hilgendorff A. Bronchopulmonary dysplasia and intrauterine growth restriction. Lancet. $2006 \mathrm{Jul}$ 1;368(9529):28.

35. Jensen EA, Edwards EM, Greenberg LT, Soll RF, Ehret DEY, Horbar JD. Severity of bronchopulmonary dysplasia among very preterm infants in the United States. Pediatrics. 2021 Jun 2;148(1):e2020030007.

\section{Funding}

Not applicable

\section{Author contribution}

\begin{tabular}{|l|l|}
\hline Rebekah Leigh & $\begin{array}{l}\text { Collect and analyze data, draft the ini- } \\
\text { tial manuscript }\end{array}$ \\
\hline John B. C. Tan & $\begin{array}{l}\text { Analyze data and critically review the } \\
\text { initial manuscript }\end{array}$ \\
\hline Shirin DeGiorgio & $\begin{array}{l}\text { Collect data, critically review the initial } \\
\text { manuscript }\end{array}$ \\
\hline Minha Cha & $\begin{array}{l}\text { Collect data, critically review the initial } \\
\text { manuscript }\end{array}$ \\
\hline Chelsea Kent & $\begin{array}{l}\text { Collect data, critically review the initial } \\
\text { manuscript }\end{array}$ \\
\hline Hung-Wen Yeh & $\begin{array}{l}\text { Interpret data and critically review the } \\
\text { initial manuscript }\end{array}$ \\
\hline Fu-Sheng Chou & $\begin{array}{l}\text { Conceptualize and design the study; } \\
\text { collect and analyze data; draft the ini- } \\
\text { tial manuscript }\end{array}$ \\
\hline
\end{tabular}

Disclosures: The authors declared no conflict of interest related to this manuscript.

NT

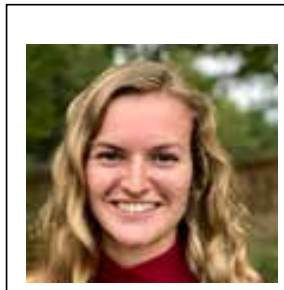

Rebekah Leigh, MS2

MD Candidate, Class of 2024

Loma Linda University School of Medicine

Loma Linda, CA

Email: rleigh@students.llu.edu 


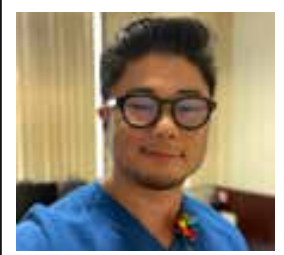

John B. C. Tan, PhD

Data Scientist

Assistant Professor of Pediatrics

Division of Neonatology, Department of Pediatrics

Loma Linda University Children's Hospital

Email: JBTan@llu.edu

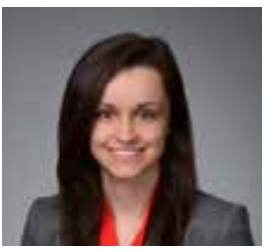

Shirin R. DeGiorgio, MD

Division of General Pediatrics and Pediatric Hospital Medicine,

Department of Pediatrics,

Loma Linda University School of Medicine,

Loma Linda, CA

Email: sdegiorgio@llu.edu

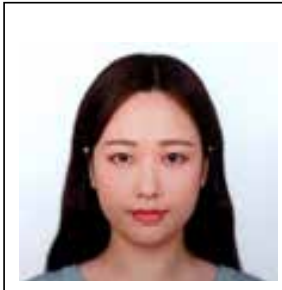

Minha Cha, BS

Medical Student Class of 2025

Loma Linda University School of Medicine

Email: mcha@students.llu.edu

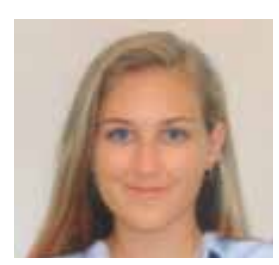

Chelsea Kent

Medical Student Class of 2025

Loma Linda University School of Medicine

Loma Linda, CA

Email: crkent@students.llu.edu

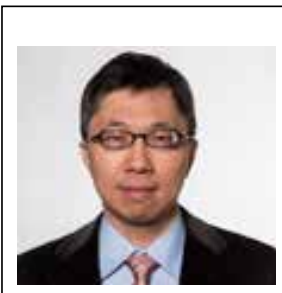

Hung-Wen Yeh, PhD

Division of Health Services and Outcomes Research,

Children's Mercy-Kansas City,

Kansas City, MO

Email: cyeh@cmh.edu

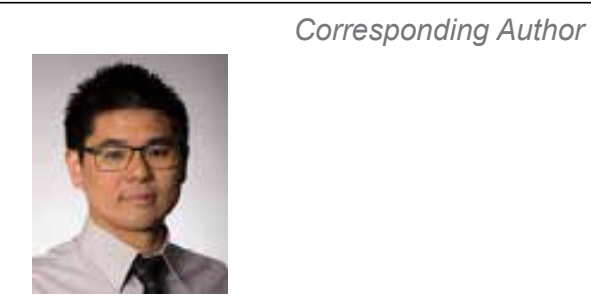

Fu-Sheng Chou, MD, PhD -

Senior Associate Editor,

Director, Digital Enterprise

Neonatology Today

Assistant Professor of Pediatrics

Division of Neonatology, Department of Pediatrics

Loma Linda University Children's Hospital

Loma Linda, CA

Email: FChou@llu.edu 